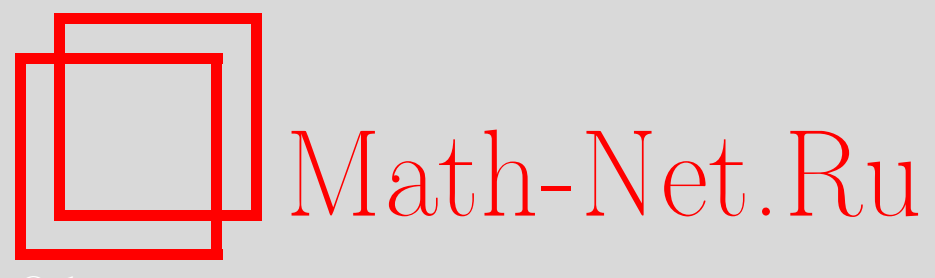

A. Даниярходжаев, Вокруг правильного пятиугольника, Квант, 2020, номер 5, 37-39

DOI: https://doi.org/10.4213/kvant20200504

Использование Общероссийского математического портала Math-Net.Ru подразумевает, что вы прочитали и согласны с пользовательским соглашением http://www . mathnet.ru/rus/agreement

Параметры загрузки:

IP : 3.81 .55 .215

26 апреля 2023 г., 13:05:42

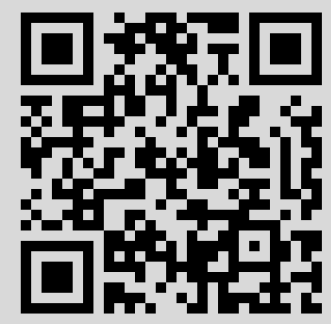


одинаковые тонкие проволочки. Для левой фигуры индуктивность равна $L_{1}, a$ для средней фигуры она равна $L_{2}$. Какова индуктивность для правой фигуры?

Пусть ток, текущий по проволочке, равен I. Обозначим поток вектора магнитной индукции, который создает один шестиугольник в самом себе, через $\Phi_{0}$, а поток, созданный им же, но в соседнем шестиугольнике, через $\Phi_{1}$. Тогда для указанных в условии задачи индуктивностей и для искомой индуктивности $L_{3}$ потоки будут такими:

$I L_{1}=3 \Phi_{0}-6 \Phi_{1}, I L_{2}=2 \Phi_{0}-2 \Phi_{1}, I L_{3}=\Phi_{0}$.

Отсюда можно найти выражение для $\Phi_{0}$ :

$$
\Phi_{0}=\frac{3 I L_{2}-I L_{1}}{3}=I\left(L_{2}-\frac{L_{1}}{3}\right),
$$

а значит, и для $L_{3}$ :

$$
L_{3}=L_{2}-\frac{L_{1}}{3} \text {. }
$$

С.Варламов

\section{ВОКРУГ ПРАВИЛЬНОГО ПЯТИУГОЛЬНИКА}

\section{А.ДАНИЯРХОДЖАЕВ}

В этой статье мы установим несколько удивительных на первый взгляд фактов о правильном пятиугольнике. В частности, дадим решение задачи М2583, опубликованной в «Кванте» №11 за 2019 год.

Пусть $a$ - сторона правильного пятиугольника, $d$ - диагональ, $h$ - расстояние между параллельными стороной и диагональю. Тогда $\frac{d}{a}=\frac{\sqrt{5}+1}{2}-$ известное $\ll 30-$ лотое сечение», а $\frac{h}{a}=\frac{\sqrt{5+\sqrt{5}}}{2 \sqrt{2}}$. Вывести эти соотношения можно из «тождеств пятиугольника»

$$
\begin{aligned}
& a^{2}+a d=d^{2}, \\
& a^{2}+d^{2}=4 h^{2},
\end{aligned}
$$

которые доказываются следующим образом. Зеркально отразим правильный пятиугольник $A B C D E$ относительно прямой $C D$ (рис.1). Из подобия треугольников $A C D$ и $A B^{\prime} E^{\prime}$ следует равносильная (1) пропорция $\frac{d}{a+d}=\frac{a}{d}$. Опустим перпендикуляр $A H$ на прямую $D E$. Так как

DOI: https://doi.org/10.4213/kvant20200504

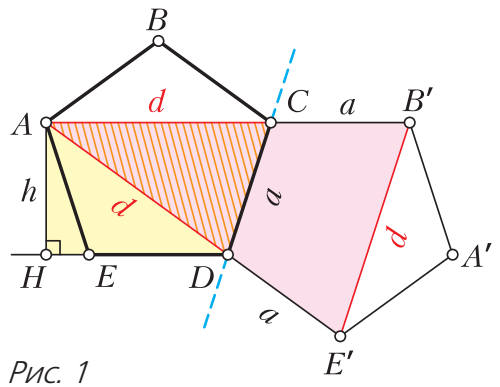

$D H=\frac{a+d}{2}$, по теореме Пифагора из треугольника $A D H$ получаем (2).

Соотношения (1) и (2), в частности, лежат в основе решения задачи М2583:

На стороне DE и диагонали ВЕ правильного пятиугольника $A B C D E$ «вовне» построены квадраты DEFG и BEHI (рис.2, a).

а) Докажите, что точки A, I и G лежат на одной прямой.

б) Докажите, что на этой же прямой лежит центр О квадрата BDJK, построенного на диагонали $B D$ «вовнутрь».

Оказывается, кроме прямой $l_{1}$, о которой идет речь в задаче, меняя направление пристраивания квадратов («вовне» или «вовнутрь»), можно получить аналогичные прямые $l_{2}$ и $l_{3}$ (рис.2,б,в).

Для доказательства рассмотрим симметричную конструкцию из четырех правильных пятиугольников (рис.3): повернем правильный пятиугольник $A B C D E$ вокруг 

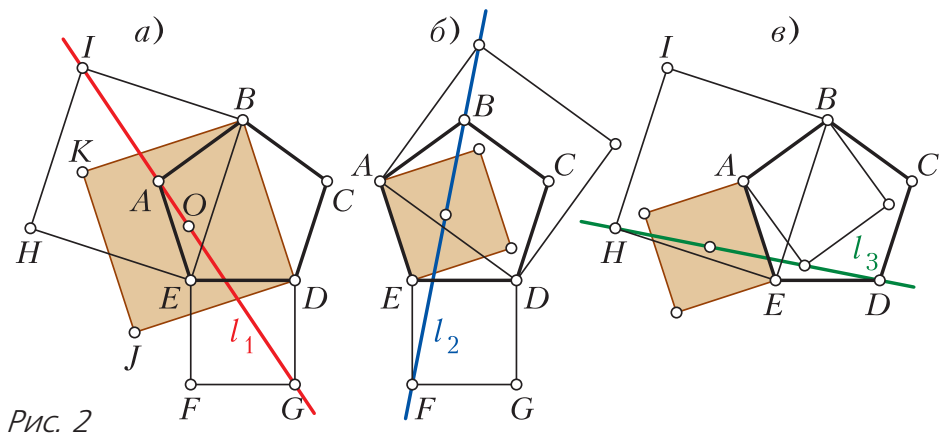

$A^{\prime} B_{2} C^{\prime} D_{1} E^{\prime}$. Очевидно, что $B D B_{2} D_{1}$ - квадрат, его центр $O$ является центром симметрии объединения четырех пятиугольников, а вершины $I$ и $G$ квадратов $B E H I$ и $D E F G$, построенных на диагонали $B E$ и стороне $D E$ «вовне», совпадают с точками $E_{1}$ и $E_{2}$. Таким образом, для решения задачи достаточно проверить, что прямоугольники $A_{1} E_{2} A_{2} E_{1}$ и

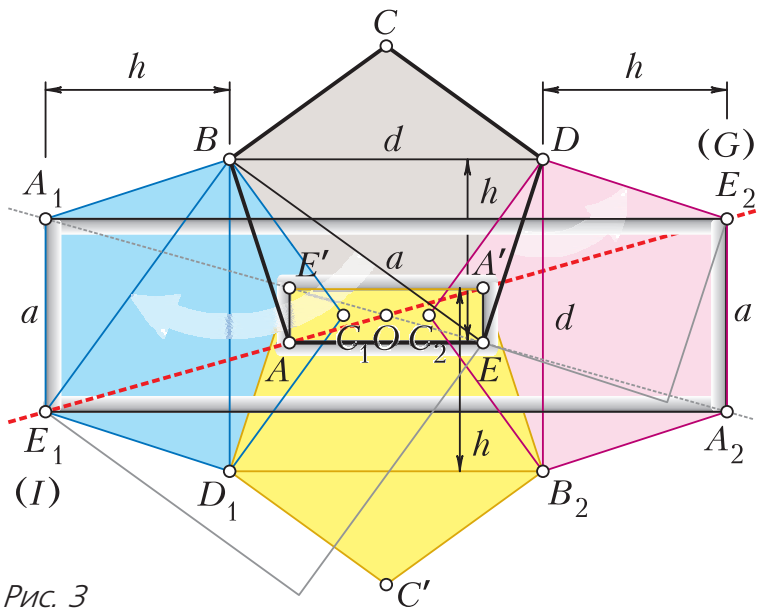

$A E^{\prime} A^{\prime} E$ (с центром $O$ и параллельными соответствующими сторонами) подобны. Их размеры: $(2 h+d) \times a \quad$ и $a \times(2 h-d)$. В силу (2) отношения соответствующих сторон равны: $(2 h+d): a=$ $=a:(2 h-d)$. Задача M2583 решена.

Используя рисунок 4 , аналогично можно доказать утверждения о прямых $l_{2}$ и $l_{3}$.

Покажем теперь, что прямые $l_{1}, l_{2}, l_{3}$ пересекаются в одной точке; $l_{2}$ и $l_{3}$ перпендикулярны; $l_{1}$ - биссектриса угла, образованного $l_{2}$ и $l_{3}$, т.е. углы между прямыми $l_{1}$ и $l_{2}, l_{1}$ и $l_{3}$ равны по $45^{\circ}$.

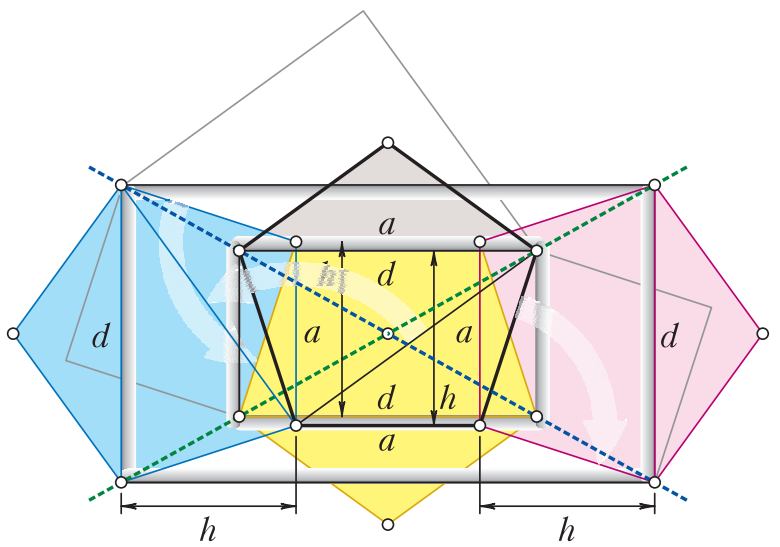

Pnc. 4

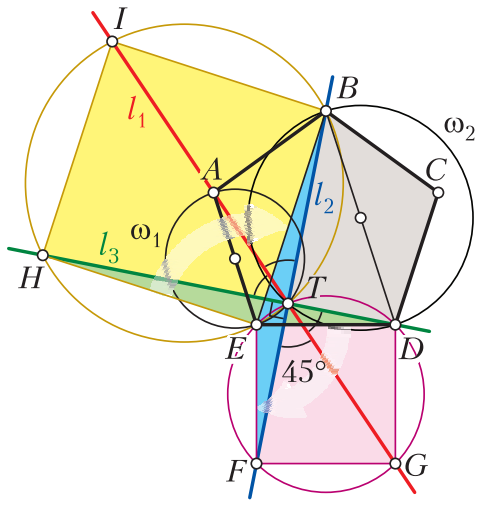

Pис. 5

точек $B$ и $D$ на $-90^{\circ}$ и $+90^{\circ}$ соответственно (считаем, что вращение против хода часовой стрелки совершается на положительные углы, а по ходу - на отрицательные); обозначим полученные пятиугольники $A_{1} B C_{1} D_{1} E_{1}$ и $A_{2} B_{2} C_{2} D E_{2}$; образ $A_{2} B_{2} C_{2} D E_{2}$ при повороте вокруг $B_{2}$ на $+90^{\circ}$ обозначим
Пусть $F B\left(l_{2}\right)$ и $H D\left(l_{3}\right)$ пересекаются в точке $T$ (рис.5). Заметим, что при повороте вокруг $E$ на $-90^{\circ}$ треугольник $H E D$ переходит в треугольник $B E F$. Значит, $H D \perp B F$. Поэтому точка $T$ лежит на описанных окружностях квадратов $D E F G$ и $B E H I$. Но тогда $T G$ - биссектриса угла 
$F T D$ (так как $F G=D G$ ), а $T I-$ биссектриса угла $B T H$ (так как $H I=B I)$. Следовательно, точки $I, T$ и $G$ лежат на одной прямой прямой $l_{1}$.

Через точку $T$ проходят не только три прямые и две окружности (описанные около квадратов), но, оказывается, еще и окружности $\omega_{1}$ и $\omega_{2}$, построенные на $A E$ и $B D$ как на диаметрах. Это не очень удивительно, так как $\angle B T D=90^{\circ}, \angle A T E=\angle A T F-$ $-\angle E T F=135^{\circ}-45^{\circ}=90^{\circ}$ (см. рис.5).

Интереснее, что $\omega_{1}$ и $\omega_{2}$ ортогональны. Напомним: углом между окружностями называют угол между касательными к ним, проведенными в точке пересечения. Пусть $P, Q, R$ - середины $A E, B D, D E$ соответственно (рис.6). Равенство (2) равносильно $P Q^{2}=P T^{2}+Q T^{2}$, т.е. $P T \perp T Q$, что и означает ортогональность $\omega_{1}$ и $\omega_{2}$. Есть и другое, «более геометрическое» доказательство ортогональности $\omega_{1}$ и $\omega_{2}$, которое, таким образом, дает еще один способ вывести (2). Поскольку $B R \perp D E$, точка $R$ лежит на $\omega_{2}$. Покажем, что точка $S$ пересечения отрезков $B P$ и $A R$ также лежит на $\omega_{2}$. При повороте вокруг центра пятиугольника на $72^{\circ}$ треу-

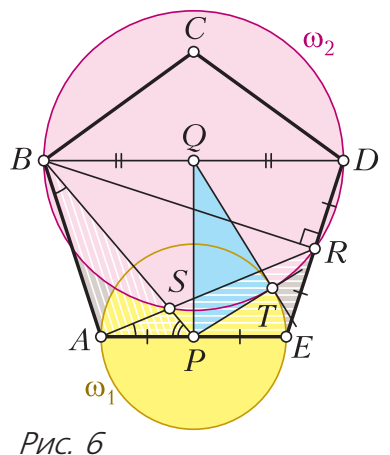

гольник $A E R$ переходит в треугольник $B A P$, значит, $\angle B S R=108^{\circ}$. А так как $\angle B D R=72^{\circ}$, четырехугольник $B D R S$ вписанный, т.е. $S \in \omega_{2}$. Заметим, что треугольники $A S P$ и $B A P$ подобны (по двум углам: $\angle P$ общий, $\angle S A P=\angle P B A$ из равенства треугольников $A E R$ и $B A P)$. Значит, $\frac{P A}{P B}=\frac{P S}{P A}$, или $P A^{2}=P S \cdot P B$. Beличина $P S \cdot P B$ - это степень точки $P$ относительно $\omega_{2}$, она равна $P Q^{2}-B Q^{2}$. Следовательно, $P T^{2}=P A^{2}=P Q^{2}-B Q^{2}=$ $=P Q^{2}-Q T^{2}$. Это и означает перпендикулярность $Q T$ и $P T$.

\section{Прыжки в воду}

Философу, физику и математику требуется решить такую задачу: прыгнуть с вышки в бассейн диаметром метр.

Ну, философ сосредоточился, вспомнил Сократа и Гегеля и, понадеявшись на удачу, прыгнул. И... не попал.

Физик измерил скорость и направление ветра, высоту вышки, все рассчитал, прыгнул и... попал.

Математик построил модель, написал программу, получил траекторию полета, долго что-то вычислял, потом разбежался, прыгнул и... улетел вверх. В знаке ошибся.

\section{Кто как может}

Инженер, физик и математик поселились в отеле. В одно и то же время у каждого в номере возникает пожар. Инженер тут же выбегает в коридор, хватает со стены пожарный шланг, открывает воду и быстро заливает огонь в своем номере. Физик мгновенно прикидывает температуру пламени, объем горючих веществ, атмосферное давление и т.д., затем наливает в стакан нужное количество воды, которой заливает очаг возгорания. Математик выскакивает в коридор, видит на стене огнетушитель, радостно восклицает «Решение существует!», после чего спокойно возвращается в номер.

\section{Перезанимался}

Студент заходит во время сессии в библиотеку.

- Где библиотекарь? - спрашивает он уборщицу.

- В архиве.

- Разархивируйте, пожалуйста, мне срочно нужна книжка.

\section{Слишком просто}

Математик идет по улице и видит афишу «Выступает камерный хор». Заинтересовавшись, покупает билет и идет на концерт. Вскоре выходит разочарованный:

- Частный случай, $k$ равно трем.

\section{Неожиданный ракурс}

На экзамене по элементарной геометрии в педвузе профессор спрашивает студента:

- Можете ли вы дать определение точки?

- Запросто. Точка - это прямая линия, если смотреть ей прямо в торец.

\section{Из книги «Математики тоже шутят»}

